\title{
Radio Network Forensic with mmWave Using the Dominant Path Algorithm
}

\author{
Usman Rauf Kamboh (D), Muhammad Rehman Shahid (D), ${ }^{1}$ Hamza Aldabbas (D), \\ Ammar Rafiq $\mathbb{D}^{3}{ }^{3}$ Bader Alouffi $\mathbb{D D}^{4}{ }^{4}$ Muhammad Asif Habib $\mathbb{D}^{5},{ }^{5}$ and Ubaid Ullah $\mathbb{D}^{1}$ \\ ${ }^{1}$ Department of Computational Sciences, The University of Faisalabad, Faisalabad 38000, Pakistan \\ ${ }^{2}$ Prince Abdullah Bin Ghazi Faculty of Information and Communication Technology, Al-Balqa Applied University, \\ Al Salt, Jordan \\ ${ }^{3}$ NFC Institute of Engineering and Fertilizer Research, Faisalabad, Pakistan \\ ${ }^{4}$ Department of Computer Science, College of Computers and Information Technology, Taif University, P.O. Box 11099, \\ Taif 21944, Saudi Arabia \\ ${ }^{5}$ Department of Computer Science, National Textile University, Faisalabad, Pakistan
}

Correspondence should be addressed to Muhammad Asif Habib; drasif@ntu.edu.pk

Received 27 August 2021; Accepted 4 December 2021; Published 12 January 2022

Academic Editor: Mamoun Alazab

Copyright (C) 2022 Usman Rauf Kamboh et al. This is an open access article distributed under the Creative Commons Attribution License, which permits unrestricted use, distribution, and reproduction in any medium, provided the original work is properly cited.

\begin{abstract}
For the last two decades, cybercrimes are growing on a daily basis. To track down cybercrimes and radio network crimes, digital forensic for radio networks provides foundations. The data transfer rate for the next-generation wireless networks would be much greater than today's network in the coming years. The fifth-generation wireless systems are considering bands beyond $6 \mathrm{GHz}$. The network design of the next-generation wireless systems depends on propagation characteristics, frequency reuse, and bandwidth variation. This article declares the channel's propagation characteristics of both line of sight (LoS) and non-LOS (NLoS) to construct and detect the path of rays coming from anomalies. The simulations were carried out to investigate the diffraction loss (DL) and frequency drop (FD). Indoor and outdoor measurements were taken with the omnidirectional circular dipole antenna with a transmitting frequency of $28 \mathrm{GHz}$ and $60 \mathrm{GHz}$ to compare the two bands of the 5th generation. Millimeter-wave communication comes with a higher constraint for implementing and deploying higher losses, low diffractions, and low signal penetrations for the mentioned two bands. For outdoor, a MATLAB built-in 3D ray tracing algorithm is used while for an indoor office environment, an in-house algorithmic simulator built using MATLAB is used to analyze the channel characteristics.
\end{abstract}

\section{Introduction}

Present research work in the field of network forensic is on traditional networks. Because of the emergence of cellular or radio networks, radio network forensic provides the platform to investigate, capture, and detect faults, illegal activities, and cybercrimes [1].

Figure 1 illustrates the investigation process for digital forensic. The DFRWS model begins from identifying the crime. This identification is further divided to different tasks, for example, signature resolving, system monitoring, analysis, profile detection, complaints, and anomalous detection.
The next step is the preservations. In preservations, we need to set up case management, management of the technology, insurance of custody chain, and synchronizing the time element. In the third step, data are collected by following the approved methodology. Examination is the fourth stage in DFWS. In examination, data are examined. Then, the data are analyzed, and tracing of evidences, validation of data, and filtering of data begin. In the presentation stage, we document the evidence, and final decision is taken. Frequencies greater than sub- $6 \mathrm{GHz}$ are not properly utilized in digital forensics due to which mmWave provides much greater bandwidth than the existing ones. On the one hand, 


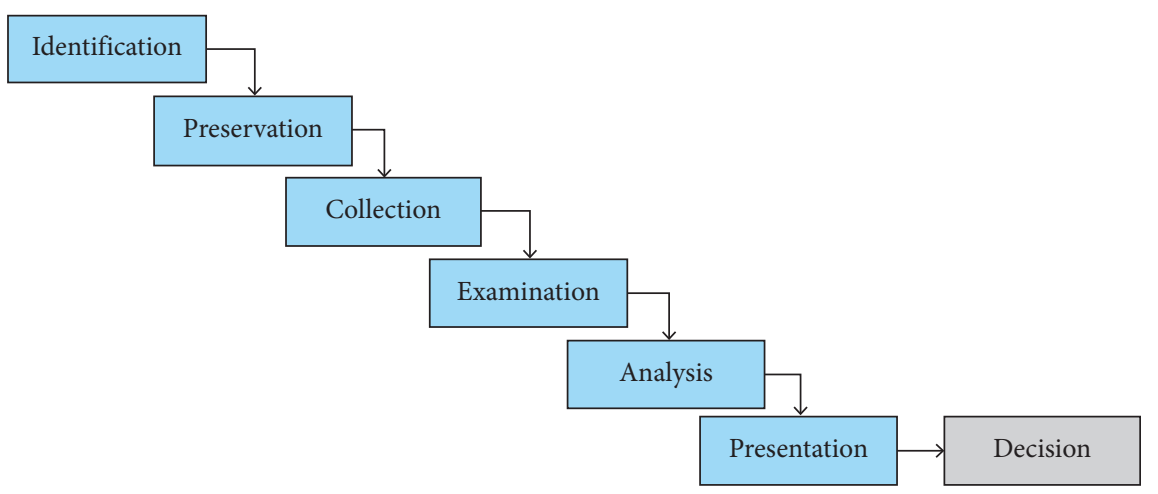

FIgURE 1: Investigative process for digital forensic science (DFRWS).

higher-frequency bands (e.g., mmWave bands) are not heavily utilized and, thus, offer larger bandwidths for wireless communication systems. Hence, determinations are focused upon the discovery of advanced frequencies as an alternate to the sub-6 GHz band [2]. On the other hand, the performance of the wireless communication system (WCS) is affected by multipath fading. As a matter of fact, the mobile device and the router for WCS have to cope up multipath manifestations which in other words demand deep knowledge of the propagation channel [3]. The designers of radio coverage make use of path loss prediction models and other channel parameters to deploy and install access points in an environment for the sake of better coverage and through put [4]. This work deals with extensive measurements, modeling, ray tracing, and simulations of channel characteristics in open outdoor as well as in narrow corridor and institution room environment. The frequency bands considered for the WCS modeling are $28 \mathrm{GHz}$ and $60 \mathrm{GHz}$. We rationally premeditated received signal strength (RSS) by the principle of the "five rays" and "two rays" receiving power model.

To associate proposed model systematic results, simulations and measurements were performed at The University of Faisalabad, Faisalabad, Pakistan. The experimental campaign is shown in Figure 2(a). The outdoor scenario is shown in Figure 2(b). The transmitter indicated in red is roughly $250 \mathrm{~m}$ apart from the receiver shown in blue. The remaining part of the article is described as follows. A summary of the recent related work and the contribution are discussed in Section 2. Section 3 deals with the path loss modeling for both outdoor as well as indoor environment. Section 4 deals with the ray tracing modeling. Section 5 analyzes the detailed simulations and algorithm for the smart ray tracing algorithm. Finally in Section 6, we draw conclusion.

\section{Recent Studies and Contributions}

Numerous tactics are suggested in the literature to control the extraordinary attenuation issue on mmWave frequency bands $[6,7]$. In $[8,9]$, the authors indicated that increment in the directivity or gain of the concerned antennas can be beneficial in high attenuation problems. Table 1 illustrates the recent findings in the field of mmWave. The model used, geometry, scenario, carrier frequency, and separation between the transmitter and receiver are mentioned against each reference. Another aspect is the material characteristics of the environment [32]. The need for ray tracing comes here as the indoor and outdoor environment difference in geometry so is the LoS and NLoS for numerous frequency bands. Ray tracing is used to model the propagation characteristics [33-36].

A separate ray source is presented in this article for endto-end penetration. We considered five rays for indoor and five for outdoor. In indoor, we analyzed one ray for LoS and the rest of four for the wall, ground, and ceiling of the room. All the five rays considered to be contributing in received and illustrated with different colors. These remain because of the small separation between transmitter and receiver antennas. The reflected rays are excluded by $\mathrm{Rx}$ because of the directivity pattern. The high fresnel reflection coefficients are organised with reference to the radio links. An escalation in the RSS was spotted as compared to outdoors. The path loss for the outdoor open environment has greater slope than that for indoor.

Normally, two ray models are deliberated for outdoors because of their nature of modeling $P_{r}$ in an unavailability scenario of $1^{\text {st }}$ order reflections. Thus, no concrete solid evidence of the difference is found between PL slopes. At last, the calculated modeling is compared with experimental parameters and RT simulations. We also prepared a reasonable investigation of the measurements with 5-ray and 2ray investigative models, and $\mathrm{RT}$ simulations with 5 rays are delivered for the indoor office environment.

In Figure 3(a), the probability of dipole antenna for the proposed model is marginal with the theoretical values. This indicates that the probability of dipole decreases with the increase in path loss. Additionally, the probability for closein and 3GPP models deviates from the theoretical model. Figure 3(b) indicates the reactance and resistance of the dipole antenna. It is evident from the graph that the 


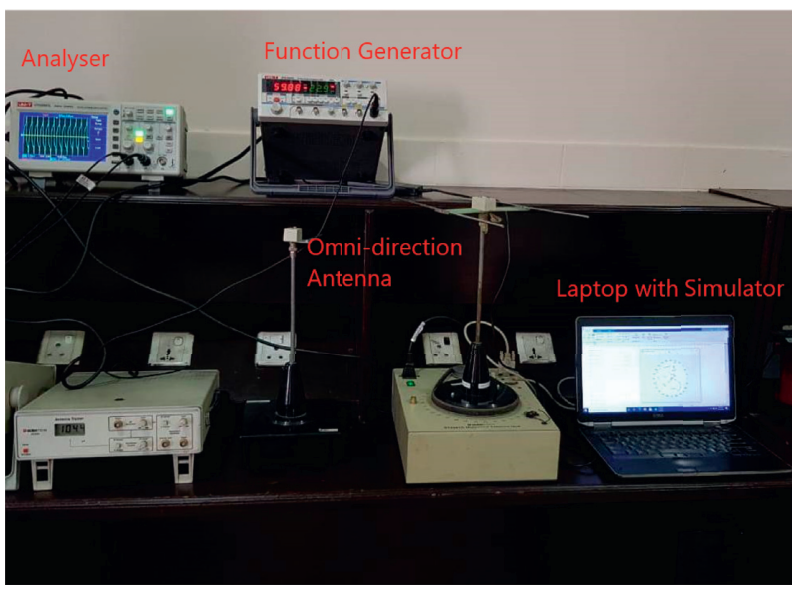

(a)

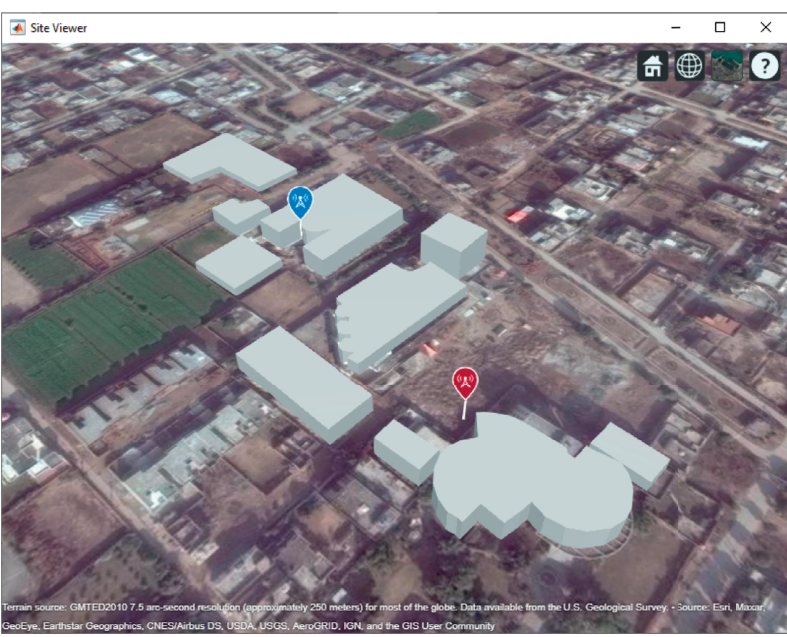

(b)

Figure 2: Measurement environment. (a) Indoor setup [5]. (b) Aerial view.

TABLE 1: Recent studies.

\begin{tabular}{|c|c|c|c|c|c|c|}
\hline Reference & Model used & Geometry & Scenario & Antenna pattern & Frequency $(\mathrm{GHz})$ & $\mathrm{Tx}-\mathrm{Rx}(\mathrm{m})$ \\
\hline [10] & Emp. & NLoS & Suburban & Direct & 28 & Unspecified \\
\hline [11] & $\mathrm{RT}$ & NLoS & $\begin{array}{l}\text { Opened } \\
\text { Squared }\end{array}$ & Unspecified & Unspecified & Unspecified \\
\hline$[12]$ & Emp. & LoS/NLoS & Indoor & Direct & $19,28,38$ & 18 \\
\hline [13] & Emp. & LoS/NLoS & Indoor & Omni & 3.5 & 20 \\
\hline [14] & Emp. & LoS & Outdoor & Direct./Omni & 3.5 & 450 \\
\hline$[15]$ & Emp. & LoS/NLoS & $\begin{array}{l}\text { Outdoor } \\
\text { Campus }\end{array}$ & Horn/Omni & 28,38 & 30 \\
\hline [16] & Emp. & LoS/NLoS & Indoor & Omni & 40 & 3 \\
\hline [17] & RT & LoS/NLoS & Indoor & Horn & 60 & Unspecified \\
\hline$[18]$ & Emp. & NLoS & $\mathrm{I} 2 \mathrm{O}$ & Dipole & $19,28,38$ & 13.5 \\
\hline [19] & Emp. & LoS/NLoS & Indoor & Horn & $3.5,28$ & 20 \\
\hline [20] & Emp. & LoS/NLoS & Indoor & Horn & 3.5 & 20 \\
\hline [21] & Emp. & LoS/NLoS & Indoor & Omni & $310,350,390$ & 0.7 \\
\hline$[22]$ & Emp. & LoS/NLoS & Outdoor & Horn & 38 & 50 \\
\hline [23] & Emp. & LoS & Indoor & Omni & $25.5,38,37.5,39.5$ & 8.5 \\
\hline$[24]$ & Emp. & LoS & Indoor & Omni & $2.4,3.52,5.8$ & 4 \\
\hline [25] & Emp. & LoS & Outdoor & Direct & Unspecified & 28 \\
\hline$[26]$ & $\mathrm{RT}$ & LoS/NLoS & $\begin{array}{l}\text { Opened } \\
\text { Squared }\end{array}$ & Unspecified & Unspecified & Unspecified \\
\hline [27] & RT & LoS & Indoor & Isotropic & 28 & Unspecified \\
\hline [28] & $7 \mathrm{RT}$ & LoS/NLoS & Indoor & Omni & 28 & Unspecified \\
\hline [29] & $\mathrm{RT}$ & LoS/NLoS & Outdoor & Omni & 28 & Unspecified \\
\hline$[30]$ & Emp. and RT & LoS & Indoor & $\begin{array}{l}\text { Emp. Horn } \\
\text { 3D Direct. }\end{array}$ & 28 & 22.7 \\
\hline$[31]$ & $3 \mathrm{D}$ RT & LoS/NLoS & Outdoor & Dipole & 28 & 488 \\
\hline This study & 3D RT & LoS/NLoS & $\begin{array}{l}\text { Outdoor } \\
\text { Campus }\end{array}$ & Omni/Horn & 28,60 & See Tables 3 and 4 \\
\hline
\end{tabular}

reactance and resistance are opposite to each other. The above recent literature when compared with our proposed work points out the following differences:

(i) Five dominant rays for indoor and two dominant rays for outdoor were adequate considering this work. (ii) The distance of rays was considered to be resolvable when compared with LoS. Smaller is the distance of resolution and the rays superimposed on one another.

(iii) At $28 \mathrm{GHz}$ and $60 \mathrm{GHz}$, a polarized dependent variable was utilized. 


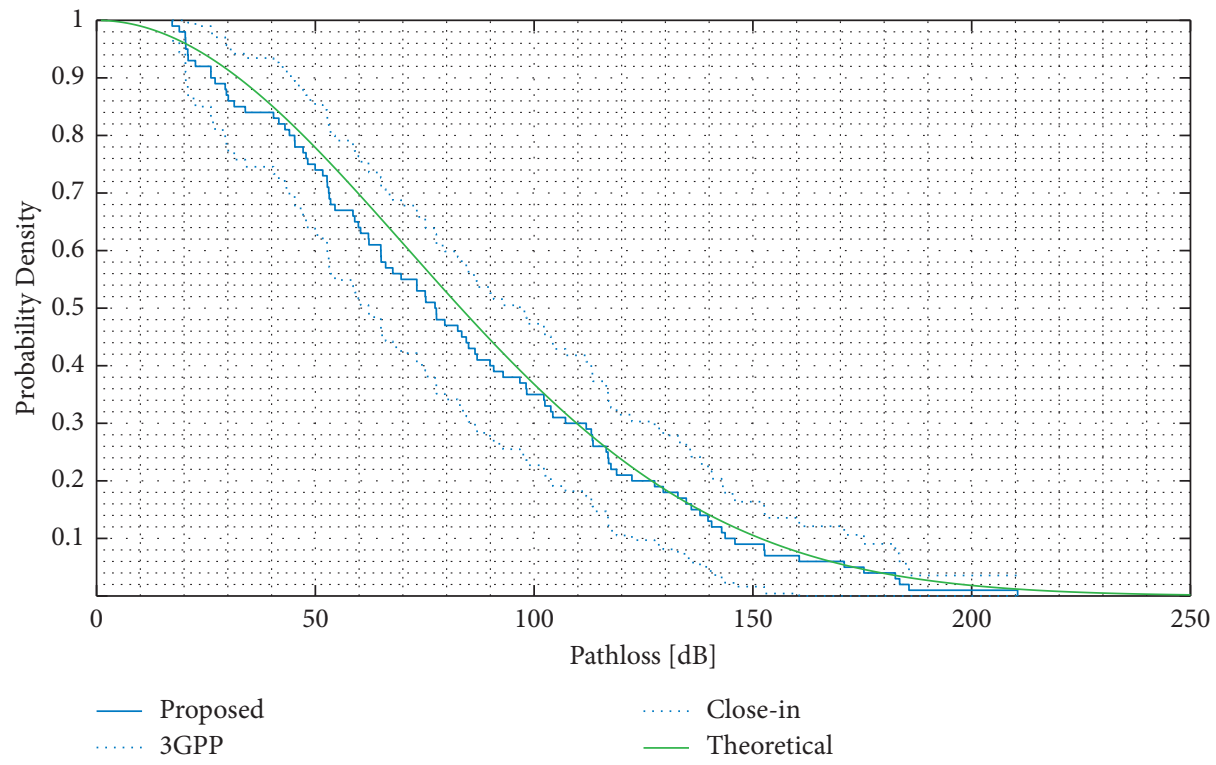

(a)

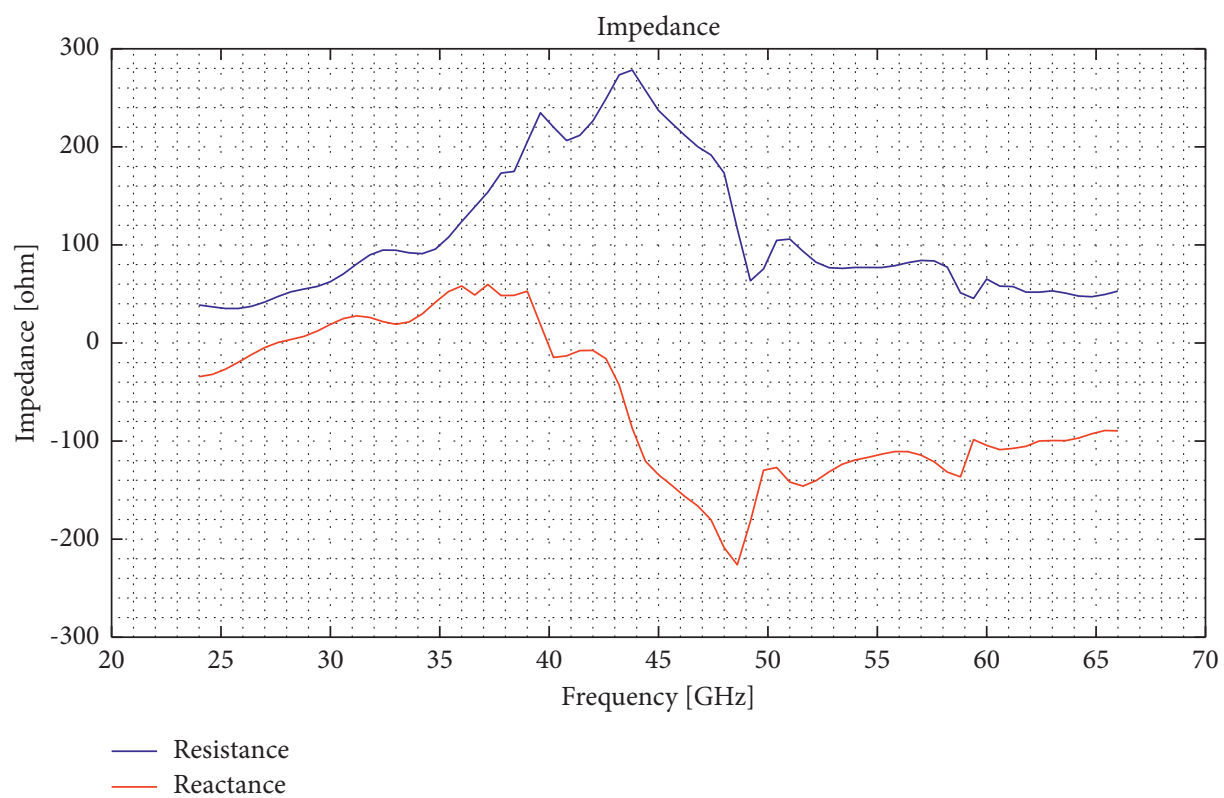

(b)

Figure 3: Circular dipole antenna characteristics. (a)Probability density of dipole antenna for different models; (b)resistance and reactance of dipole antenna.

(iv) The close deployment of Tx and Rx is very common in indoor scenarios nowadays. This scenario with a typical classroom environment is focused here. (v) Antenna gains for $\mathrm{Tx}$ and $\mathrm{Rx}$ are closely monitored with the help of MATLAB simulations using antenna radiation patterns. 


\section{Path Loss Modeling}

Figure 4 is taken from the recent studies conducted by authors to ray trace the path loss in a different environment with heatmap techniques using MATLAB Simulink. The close-in (CI) free-space path loss model is utilized to adjust the path loss from the result of simulations. The supremacy of CI is that its parameters are dependent on the operating frequency. The relationship of PL with frequency for CI is as follows [37]:

$$
\mathrm{PL}^{\mathrm{CI}}(d)[\mathrm{d} B]=\operatorname{FSPL}\left(f, d_{0}\right)+10 n \cdot \log \left(\frac{d}{d_{0}}\right)+X_{\sigma_{\mathrm{SF}}}^{\mathrm{CI}} .
$$

$X_{\sigma_{\mathrm{SF}}}^{\mathrm{CI}}$ denotes the standard deviation. $\operatorname{FSPL}\left(f, d_{0}\right)$ represents the free-space path loss in $\mathrm{dB}$ and is given by

$$
\operatorname{FSPL}\left(f, d_{0}\right)=20 \log 10\left(\frac{\left(4 \pi d_{0} f\right)}{c}\right)
$$

where $d_{0}$ is the Tx-Rx separation at $1 m$ reference; $f$ stands for the frequency of the carrier radio wave; $c$ denotes the light speed, and $n$ is the path loss exponent (PLE). In [38], WINNER II and 3GPP are illustrated with the help of floating intercept (FI). According to [38], FI is expressed as follows:

$$
\mathrm{PL}^{\mathrm{FI}}(d)[d B]=\alpha+10 \beta \log _{10}(d)+Y_{\sigma_{\mathrm{SF}}}^{\mathrm{FI}},
$$

where $\sigma_{\mathrm{SF}}$ of $Y_{\sigma_{\mathrm{SF}}}^{\mathrm{FI}}$ is the Gaussian SF. Multifrequency path loss models are used for a variety of mmWaves. For this aspect, the famous alpha-beta-gamma (ABG) model with a $1 \mathrm{~m}$ reference distance and $1 \mathrm{GHz}$ referred frequency is considered here as follows [39]:

$$
\begin{aligned}
\mathrm{PL}^{\mathrm{ABG}}(f, d)(\mathrm{dB})= & 10 \alpha \cdot \log \left(\frac{d}{d_{0}}\right)+10 \gamma \cdot \log \left(\frac{f}{f_{\text {ref }}}\right) \\
& +\beta+X_{\sigma}^{\mathrm{ABG}}
\end{aligned}
$$

In the above equation, $\alpha$ and $\gamma$ denote constant coefficients. $\beta$ denotes the offset, and $X_{\sigma}^{\mathrm{ABG}}$ is a Gaussian random variable. In literature [40], ABG is solved with the help of MMSE to minimize error.

Another derived model from CI and FSPL which is widely used for path loss analysis is CIF (Close in free space) [38]:

$\mathrm{PL}^{\mathrm{CIF}}(f, d)[\mathrm{d} B]=10 m \cdot \log _{10}\left(d_{0}\right) \cdot\left(1+g \cdot \frac{f-f_{0}}{f_{0}}\right)+X_{\sigma}^{\mathrm{CIF}}$,

where $m$ and $g$ are the distance dependent and linear frequency dependent factors, respectively.

\section{RT Modeling}

Ray tracing involves two patterns. (a) ray launch and (b) the image theory. In the first line of attack, rays commencing $\mathrm{Tx}$ are launched with even angular departure and then traced. After tracing the rays, the received field will be calculated to find the power [41-43]. This approach has faster computational time but has less accuracy when the separation increases. An alternate approach as mentioned is the image method, in which the route is first defined of image points from $\mathrm{Tx}$ or $\mathrm{Rx}[41,43]$. This method of images has a drawback of high computational time and involves complex algorithms. The interaction mechanism implements propagation's theory; nonetheless, the deviation through different RT tools occurs due to the dissimilar method of modeling the similar phenomena (e.g., diffraction, diffusion, scattering, and reflection). Other deviations follow in the antenna design, pattern, sources, frequencies, and models. The constitutive material aspects, e.g., the magnetic permeability $(\mu)$, electrical permittivity $(\epsilon)$, and the conductivity $(\rho)$, are essentials for accomplishing accurate channel estimations in RT [44]. Undeniably, Maxwell's electromagnetic wave equations for diverse edges are fighting fit for constitutive parameters. This directly affects the wave's phase and amplitude, therefore, eventually governs the influence of discrete multipath constituents. The influence of "material properties" on the RT estimations precision depends on the dominant propagation.

Accordingly, in situations wherever the LoS dominates, material properties can affect very little on the accuracy of the prediction algorithm. The modeling of diffused scatterings is usually carried out with the help of severe roughness models as proposed in literature [45]. Because the model is not dependent on the Maxwell equation, it solves the issue of diffused component's power. Besides, the roughness model is not dependent on the material properties; it can predict a diffuse scattered field. Fresnel's equation works on smooth surfaces for the analysis of reflection coefficients and transmission coefficients. The reflection coefficient for the smooth surface is given by [32]

$$
\begin{aligned}
\tau_{\perp} & =\frac{e_{\perp}^{r}}{e_{\perp}^{i}} \\
& =\frac{\eta_{1} \operatorname{Sec} \theta_{t}-\eta_{0} \operatorname{Sec} \theta_{t}}{\eta_{1} \operatorname{Sec} \theta_{t}+\eta_{0} \operatorname{Sec} \theta_{t}},
\end{aligned}
$$

In the above equation, $e_{\perp}^{i}$ denotes the incident electric fields, $\theta_{i}$ represents the incident angles, $e_{\perp}^{r}$ expresses the reflected electric fields, $\eta$ is the wave impedance, and $\theta_{r}$ declares the transmission's angle. The transmission coefficients are denoted by

$$
\begin{aligned}
& t_{\perp}=\frac{e_{\perp}^{t}}{e_{\perp}^{i}}=\frac{2 \eta_{1} \sec \theta_{t}}{\eta_{1} \sec \theta_{i}+\eta_{0} \sec \theta_{t}}, \\
& t_{\|}=\frac{e_{\|}^{t}}{e_{\|}^{i}}=\frac{2 \eta_{1} \sec \theta_{t}}{\eta_{0} \sec \theta_{i}+\eta_{1} \sec \theta_{t}} .
\end{aligned}
$$

The constitutive parameters of the used materials will determine the impedance of the wave $(\eta)$ and the also the angle of transmission $\theta_{t}$ : 


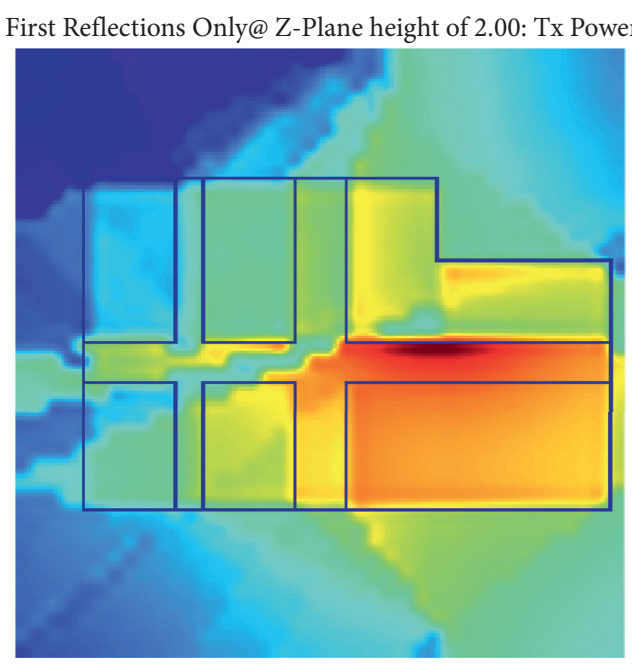

(a)

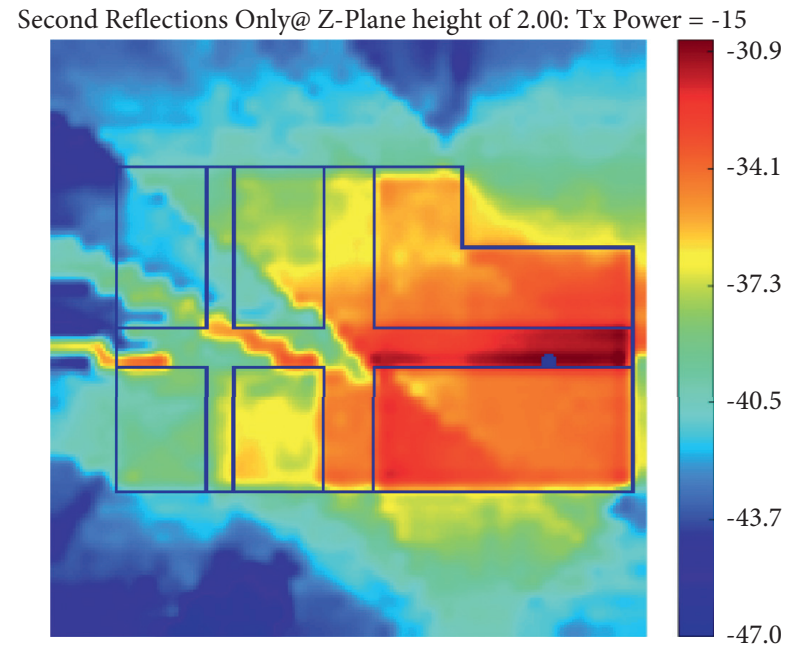

(b)

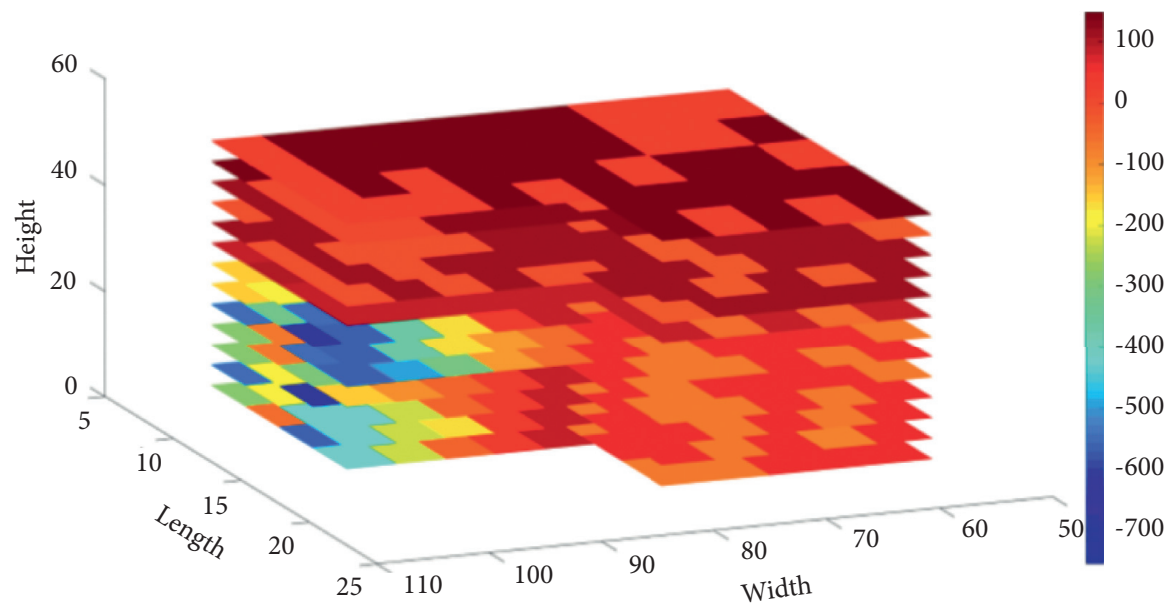

(c)

FIGURE 4: Recent related work [32]. (a) 1st reflection; (b) 2nd reflection; (c) heap map of outdoor simulations.

$$
\begin{aligned}
& \eta=\sqrt{\frac{\iota \Omega \mu}{\rho+\iota \Omega \epsilon}}, \\
& \theta_{r}=\cos ^{-1} \sqrt{1-\left(\frac{p_{0}}{p_{1}}\right)^{2}\left(\sin \theta_{t}\right)^{2}},
\end{aligned}
$$

where $\Omega$ stands for the angular frequency and $p$ denotes the wave number for that frequency.

$$
\begin{aligned}
& \Omega=2 \pi f, \\
& p=\Omega \sqrt{\mu \epsilon-\frac{\mu \mu \rho}{\Omega}} .
\end{aligned}
$$

More accurate results for diffracted wedges can be obtained by using the "Uniform Theory of Diffraction" (UTD). For UTD, the details of material constitutive parameter are necessary to be determined. Considering Figure 5, the diffraction coefficient can be obtained by [46]

$$
D_{\|}^{\perp}=\frac{-e^{-\imath \pi / 4}}{2 * p \sqrt{2 \pi p}} \times\left\{D_{i_{1}}+D_{i_{2}}+t_{\|, 0}^{\perp} D_{i_{3}}+t_{\|, p}^{\perp} D_{i_{4}}\right\}
$$

where

$$
\begin{aligned}
& D_{i_{1}}=\frac{1}{\tan \left(\pi+\left(\Psi-\Psi^{\prime}\right) / 2 p \cdot Y\left(v L b^{+}\left(\Psi-\Psi^{\prime}\right)\right)\right.}, \\
& D_{i_{2}}=\frac{1}{\tan \left(\pi-\left(\Psi-\Psi^{\prime}\right) / 2 p \cdot Y\left(v L b^{-}\left(\Psi-\Psi^{\prime}\right)\right)\right.}, \\
& D_{i_{3}}=\frac{1}{\tan \left(\pi-\left(\Psi-\Psi^{\prime}\right) / 2 p \cdot Y\left(v L b^{-}\left(\Psi-\Psi^{\prime}\right)\right)\right.}, \\
& D_{i_{4}}=\frac{1}{\tan \left(\pi-\left(\Psi-\Psi^{\prime}\right) / 2 p \cdot Y\left(v L b^{+}\left(\Psi-\Psi^{\prime}\right)\right)\right.},
\end{aligned}
$$

where $\Psi$ ' is the incidence angle and $\Psi$ is the diffraction angle; the Fresnel integral $Y(v)$ can be expressed as follows: 


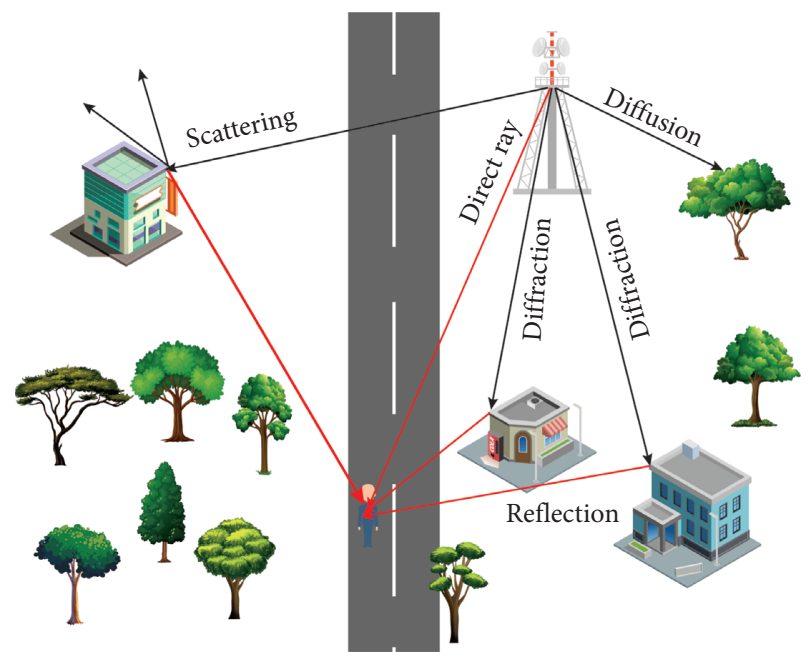

Figure 5: Dominant ray tracing concept [32].

$$
Y(v)=2 j \sqrt{(v) e^{\iota v}} \int_{\sqrt{v}}^{\infty} e^{-l \tau^{2}}
$$

$L$ is defined as follows:

$$
L=\frac{s s^{\prime}}{s+s^{\prime}}
$$

Figure 5 explains that $n$ represents the wedge's factor, and $s$ and $s^{\prime}$ are the distances. $a^{ \pm}$is defined as follows:

$a^{ \pm}\left(\Psi \pm \Psi^{\prime}\right)=2 \times\left(1-\sin ^{2}\left(\frac{2 n \Psi M^{ \pm}-\left(\Psi \pm \Psi^{\prime}\right)}{2}\right)\right)$.

Finally, $M^{ \pm}$are the integers of the following equations:

$$
\begin{aligned}
& 2 n \pi M^{+}-\left(\Psi \pm \Psi^{\prime}\right)=\pi, \\
& 2 n \pi M^{-}-\left(\Psi \pm \Psi^{\prime}\right)=-\pi .
\end{aligned}
$$

The information of material properties is hence crucial to precisely model the diffraction.

Figure 6 indicates the properties of different materials and diffraction loss occuring due to different material constitutes.

\section{Simulations with the Dominant 3D Ray Tracing}

In mmWaves (high frequencies), the concept of ray optics is considered. Maxwell equations are solved asymptotically. The wave length of the electromagnetic wave plays a key role in obtaining attractive and accurate results. Greater the size of the wavelength, the more accurate the results from an RT stimulation $[47,48]$. Reliant on the electrogeometrical appearances available in digital maps, a ray undergoes diffuse scattering, diffraction, penetration, reflection, or attenuation. The penetration of rays joints upon accurate geometric and materialistic characteristics.

The simulation setup is shown in Table 2. We have considered a three-floored building. The path loss model we used for simulations is Close in. Two mmWave frequencies
(28 GHz and $60 \mathrm{GHz}$ are discussed for the complex layout of LoS and NLoS. The length and width of the wall are $2.8 \mathrm{~m}$ and $0.1 \mathrm{~m}$, respectively. The rest of the parameters of the Base station and User terminal are mentioned in Table 2.

RT tool complications increase with the order of interaction mechanism. Nevertheless, with high order interaction, the expansions in relation to prediction precision are frequently negligible but even so acquire major computations overhead [49].

5.1. Simulations for Outdoor. The simulations performed in this campaign are based on the intelligent $3 \mathrm{D}$ ray tracer built-in MATLAB. This tool is different from other 3D RT tools as it is capable of performing full $3 \mathrm{D}$ ray tracing in terms of path loss and received power. Other ray-tracing tools can find many paths, but the mentioned tool is used to find the dominant paths. The 3D map of the concerned scenario is shown in Figure 7.

In this section, received signal strength (RSS) is the main parameter in designing the simulator. The antenna designed for outdoor simulations is an omni-directional circular dipole antenna with a transmitting frequency of $28 \mathrm{GHz}$ and $60 \mathrm{GHz}$.

Figure $8(a)$ shows the current distribution of the antenna. The current at the center (circular) is greater than that at the bottom and top of the antenna. This is because of the feed which is at the center of the antenna. Figure 8(b) shows the $3 \mathrm{D}$ radiation pattern. The maximum radiation is $1.71 \mathrm{dBi}$ and minimum is $13.2 \mathrm{dBi}$ (Algorithm 1).

The height of the receiver (user with mobile phone) is $1.6 \mathrm{~m}$. The user is standing still on the location mentioned in Table 2. Figure 7 indicates the RSS in $\mathrm{dBm}$. Figure 7(a) shows RSS for $28 \mathrm{GHz}$. It is evident that the fig that the received power at $\mathrm{Rx}$ is about $-50 \mathrm{dBm}$. Figure $7(\mathrm{~b})$ shows RSS for $28 \mathrm{GHz}$. It is evident from the figure that the received power at $\mathrm{Rx}$ is about $-40 \mathrm{dBm}$. The comparison of Figures $7(\mathrm{a})$ and 7 (b) reveal that the RSS level has a mean difference of around $8 \mathrm{dBm}-10 \mathrm{dBm}$ between $28 \mathrm{GHz}$ and $60 \mathrm{GHz}$. This is due to the fact that the huge gap between mmWave frequencies does not affect much power of the signal in small cell environments. On the other hand, this is the sign of low penetration, low signal diffraction, and very high free-space losses.

5.2. Simulations for Indoor. The indoor floor map of the simulation environment is shown in Figure 9. There are three transmitters in the facility. To understand the complex scenario for ray tracing, we choose the nearby transmitter, (in the corridor) (i.e., $\mathrm{Tx} 2$ ). $\mathrm{Rx}$ is in the office. Figure 10 illustrates the typical classroom where measurements were performed, and simulations were carried out. It consists of plywood, concrete tiles, plastic, brick work, plaster, and metal. Figure 6 shows the path loss of different material obstacles in the path of rays against a range of frequencies. It is evident that the loss due to bricks is constant throughout the range of mmWave frequencies (Algorithm 2).

Figures 11(a) and Figure 11(b) show the results of indoor simulations at $28 \mathrm{GHz}$ and $60 \mathrm{GHz}$, respectively. Five 


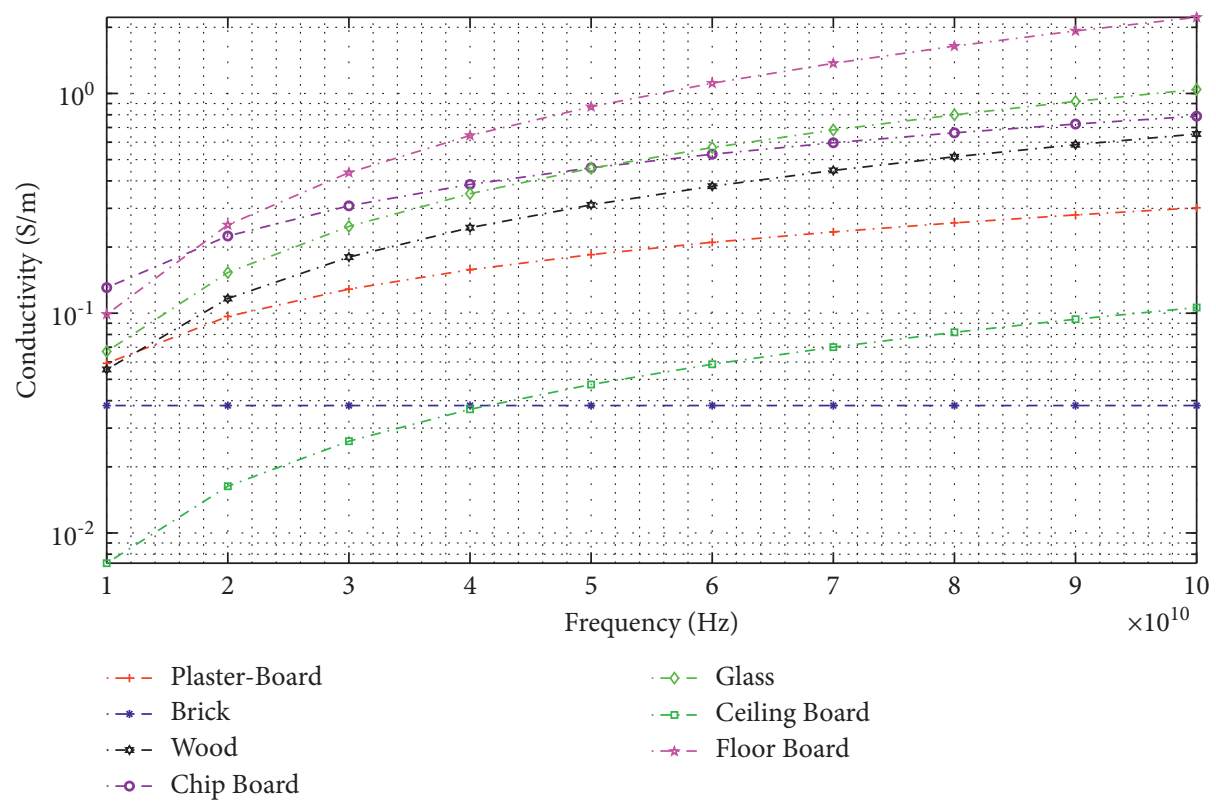

FIgURE 6: Permittivity of different materials.

TABle 2: Simulation setup.

\begin{tabular}{|c|c|c|c|}
\hline Parameter & Description & & Unit value \\
\hline Number of floors & & 3 & \\
\hline Cable losses $(\mathrm{dBm})$ & & 3 & \\
\hline Ray tracing method & & Close-in & \\
\hline Propagation model & & Full 3D & \\
\hline Coupling losses (dB) & & 70 & \\
\hline Operating frequency & mmWave & & 28 and $60 \mathrm{GHz}$ \\
\hline Layout & Complex & & LoS/NLoS \\
\hline Fading & Model & & Rayleigh \\
\hline Cell structure & Hex (grid) & & 1 \\
\hline \multirow{2}{*}{ Building's wall } & Length (m) & & 2.8 \\
\hline & Width $(\mathrm{m})$ & & 0.1 \\
\hline \multirow{6}{*}{ Base station } & Transmitting power $(\mathrm{dBm})$ & & 21.0 \\
\hline & Height $(\mathrm{m})$ & & 25.1 \\
\hline & Pattern & & Dipole \\
\hline & Bandwidth (GHz) & & 0.1 \\
\hline & Obstacle & & Enabled \\
\hline & Polarization & & Latitude \\
\hline \multirow{6}{*}{ User terminal } & Receiving power loss (dBm) & & 2 \\
\hline & Longitude $(\mathrm{m})$ & & 2.7 \\
\hline & Pattern & & Omni \\
\hline & Bandwidth $(\mathrm{GHz})$ & & 0.1 \\
\hline & Obstacle & & Enabled \\
\hline & Polarization & & Longitude/latitude \\
\hline
\end{tabular}

dominant rays are considered here to decrease computational time and make the algorithm less complex. In Figure 11(a), the color of two LoS rays is dark blue showing that the path loss is near to $80 \mathrm{~dB}$. The other rays colored red and yellow are indicating path loss of 90 and $95+$ respectively. This is due to the NLoS, reflection and diffraction of metal and brick walls. More detailed analyses of the rays and pattern of the path loss are explained in Table 3.

In Figure 11(b), the color of two LoS rays is light blue showing that path loss is near to $90 \mathrm{~dB}$. The other rays colored green and yellow indicate the path loss of 95 and 


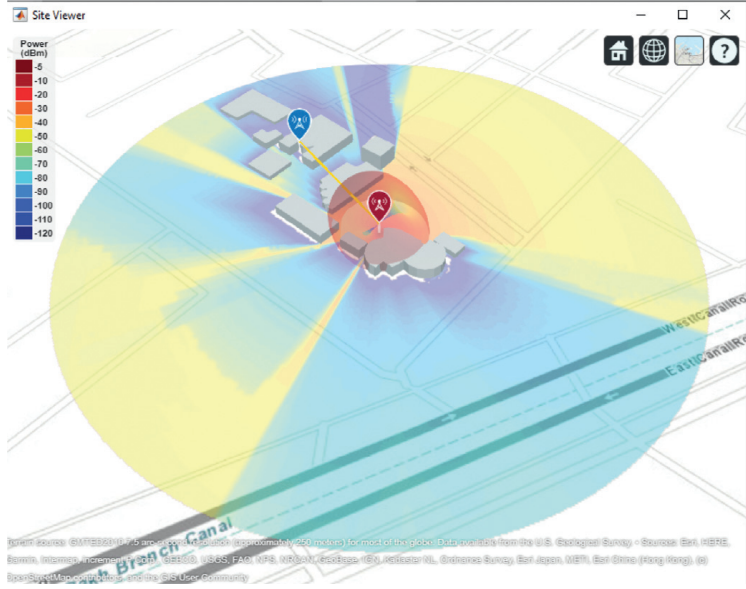

(a)

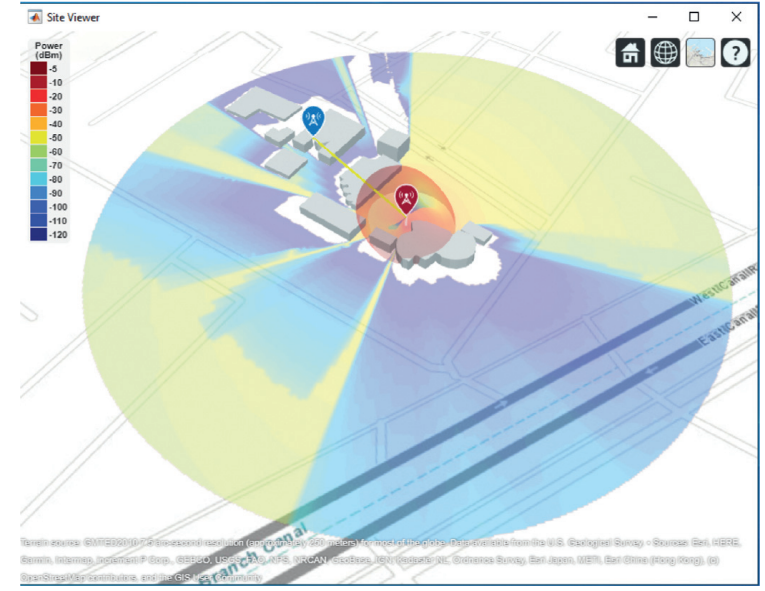

(b)

Figure 7: Coverage map. (a) At $28 \mathrm{GHz}$; (b) at $60 \mathrm{GHz}$.

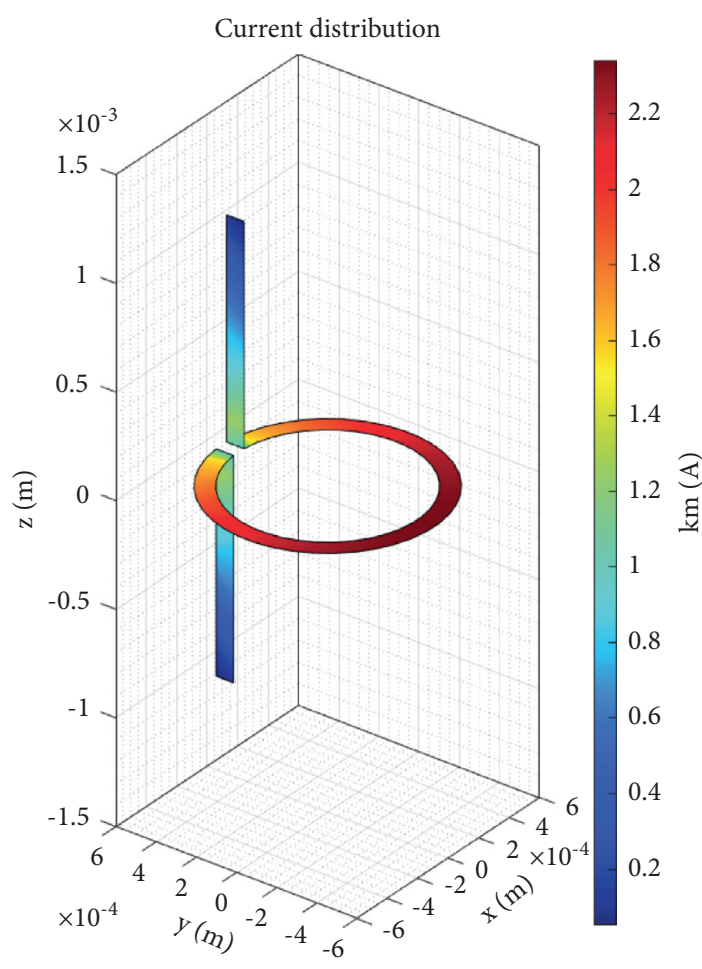

(a)

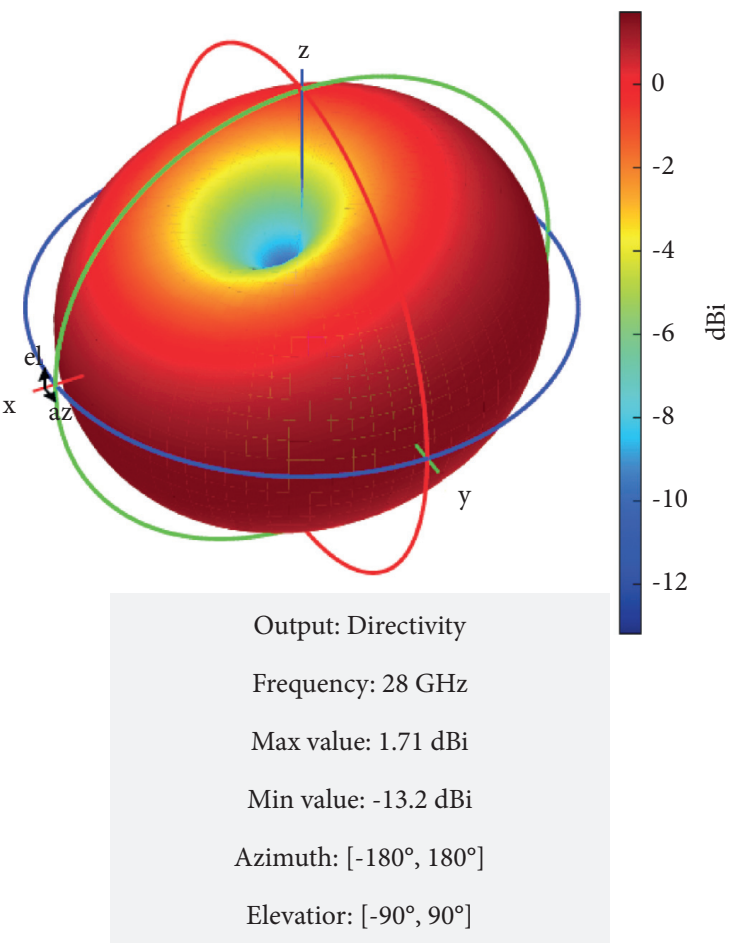

(b)

FIgURE 8: Antenna characteristics. (a) Current distribution; (b) antenna radiation pattern.

$100+$, respectively. This is due to NLoS, reflection, and diffraction of metal and brick walls. More detailed analyses of the rays and pattern of the path loss are explained in Table 4 . The difference between the path loss due to the two different transmitting frequencies is promising in a way that increasing the frequency in indoor environment results in rising the path loss by $5 \mathrm{~dB}$ for every $10 \mathrm{GHz}$. This is also clear from CI model calculations. 


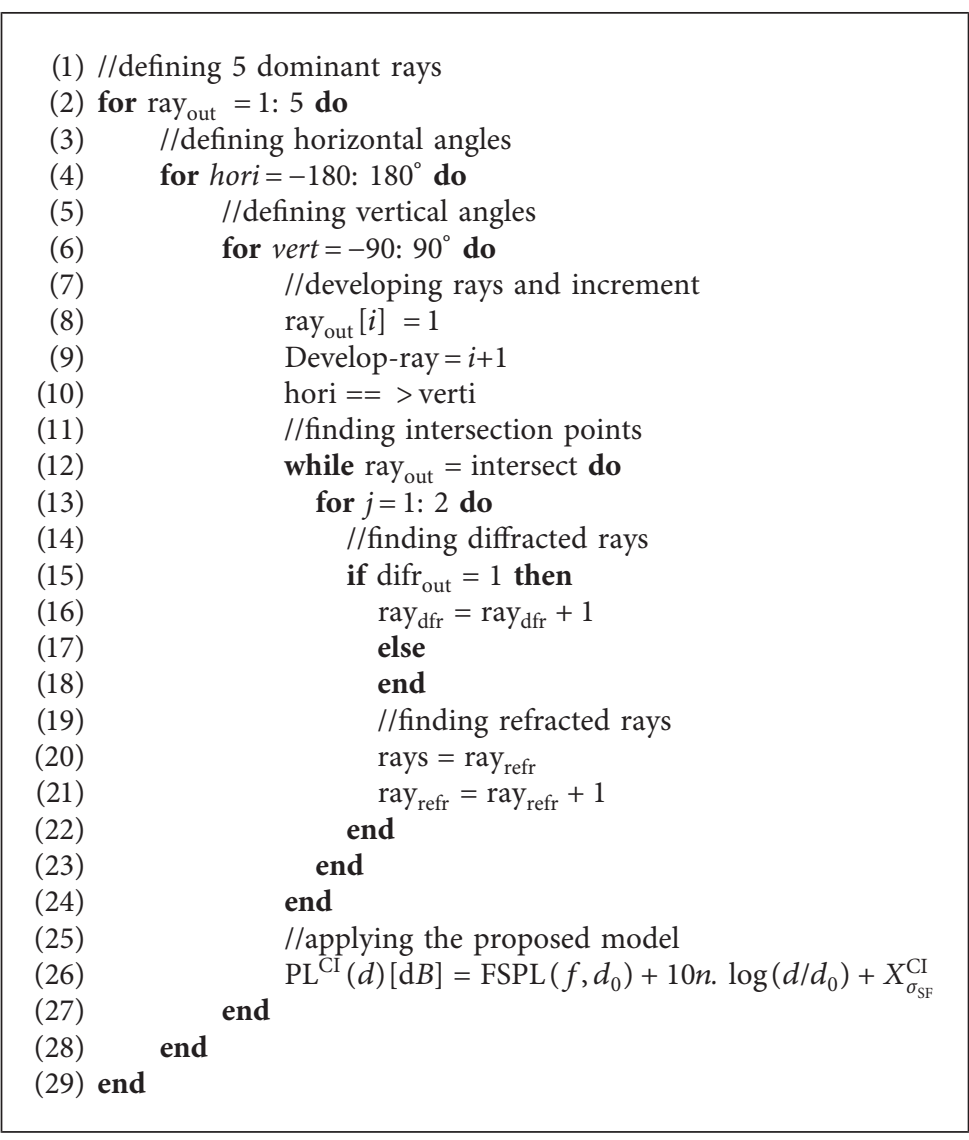

Algorithm 1: Outdoor ray-tracing algorithm pseudocode.

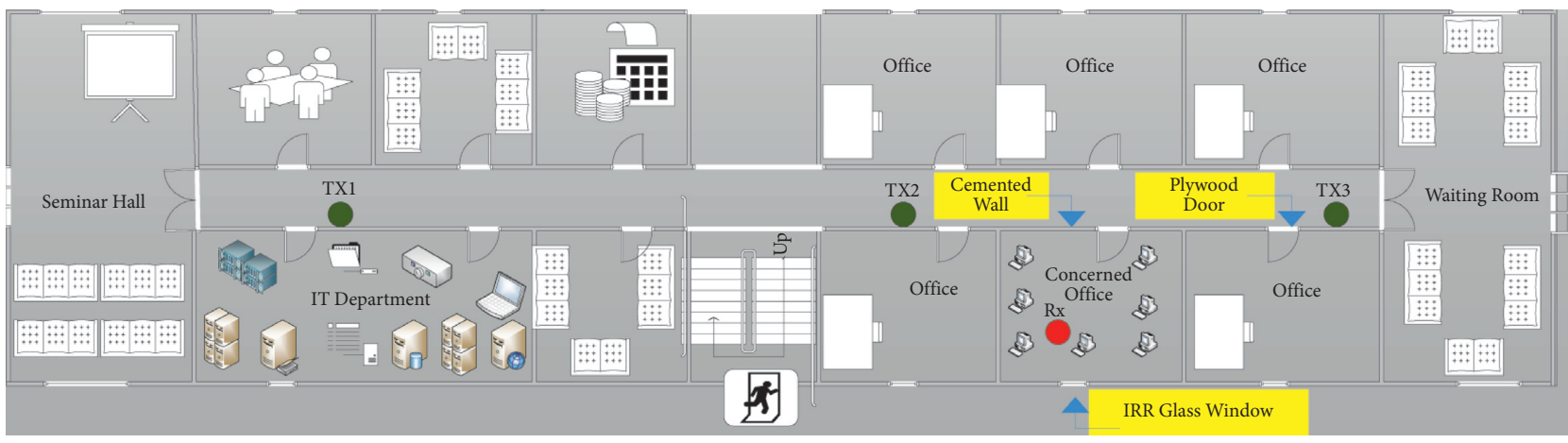

Figure 9: Floor plan for indoor simulations [5].

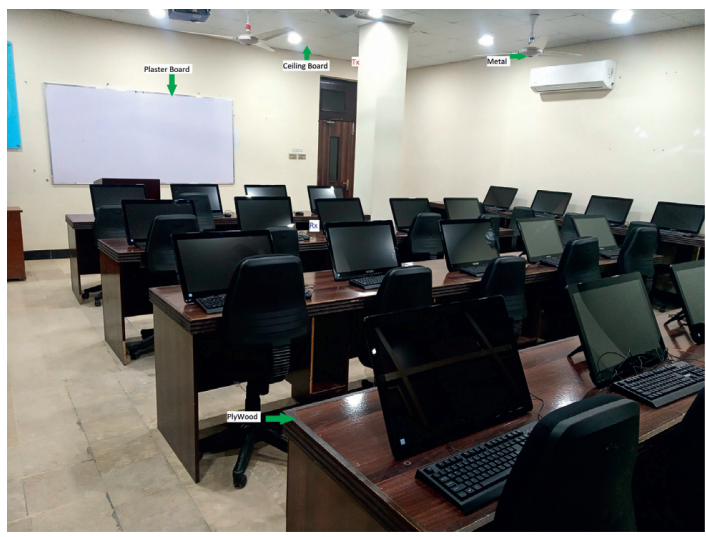

Figure 10: Typical classroom under consideration. 


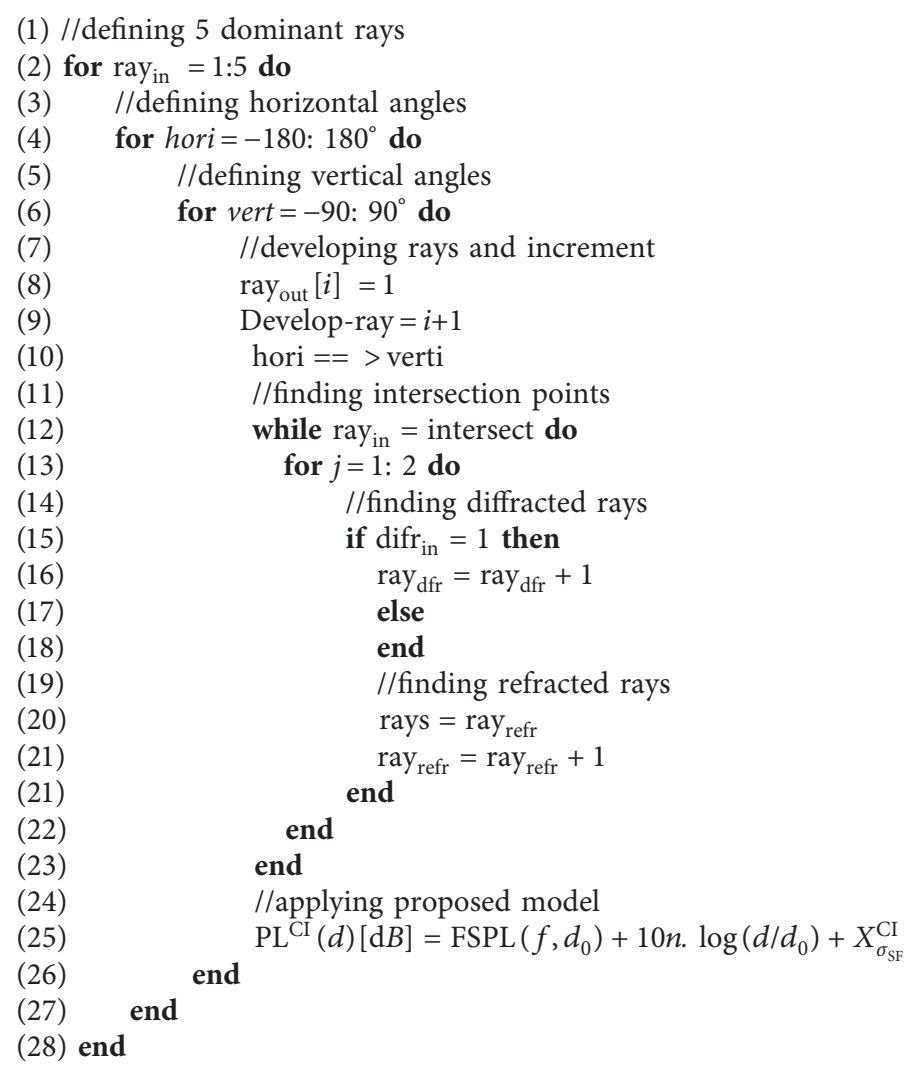

Algorithm 2: Indoor ray-tracing algorithm pseudocode.

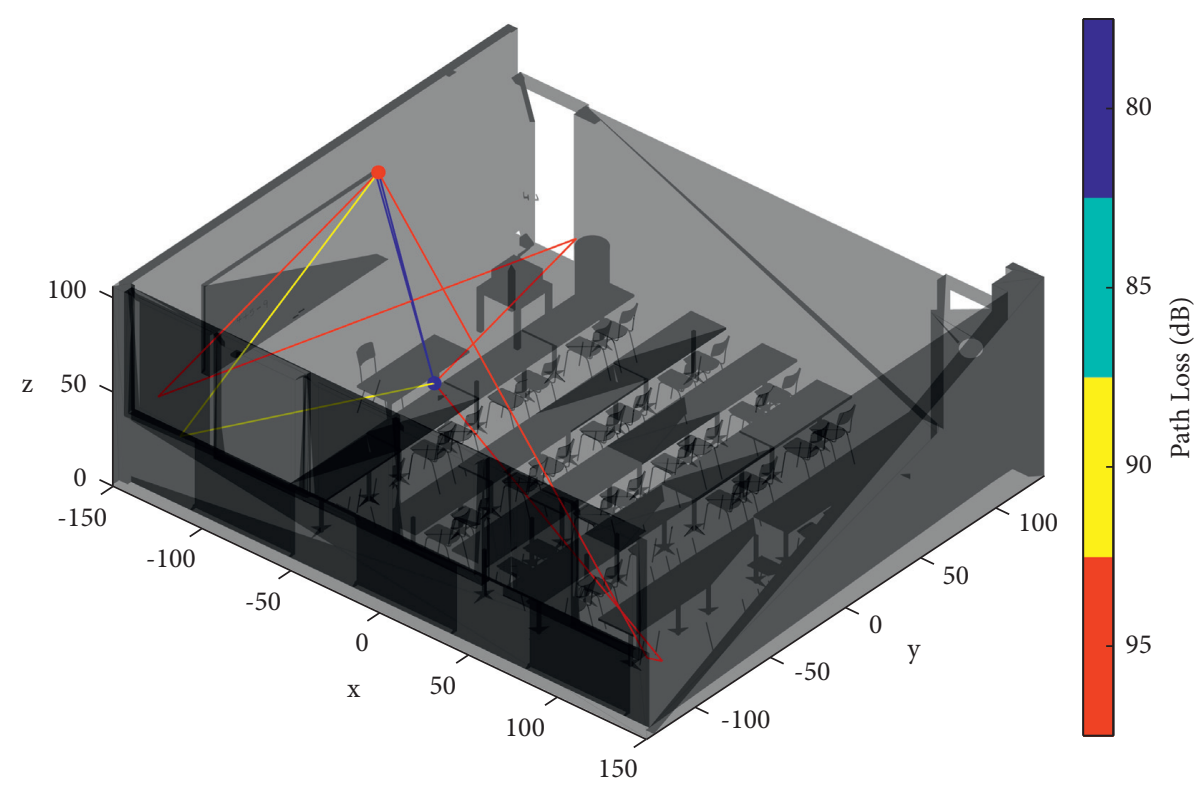

(a)

Figure 11: Continued. 


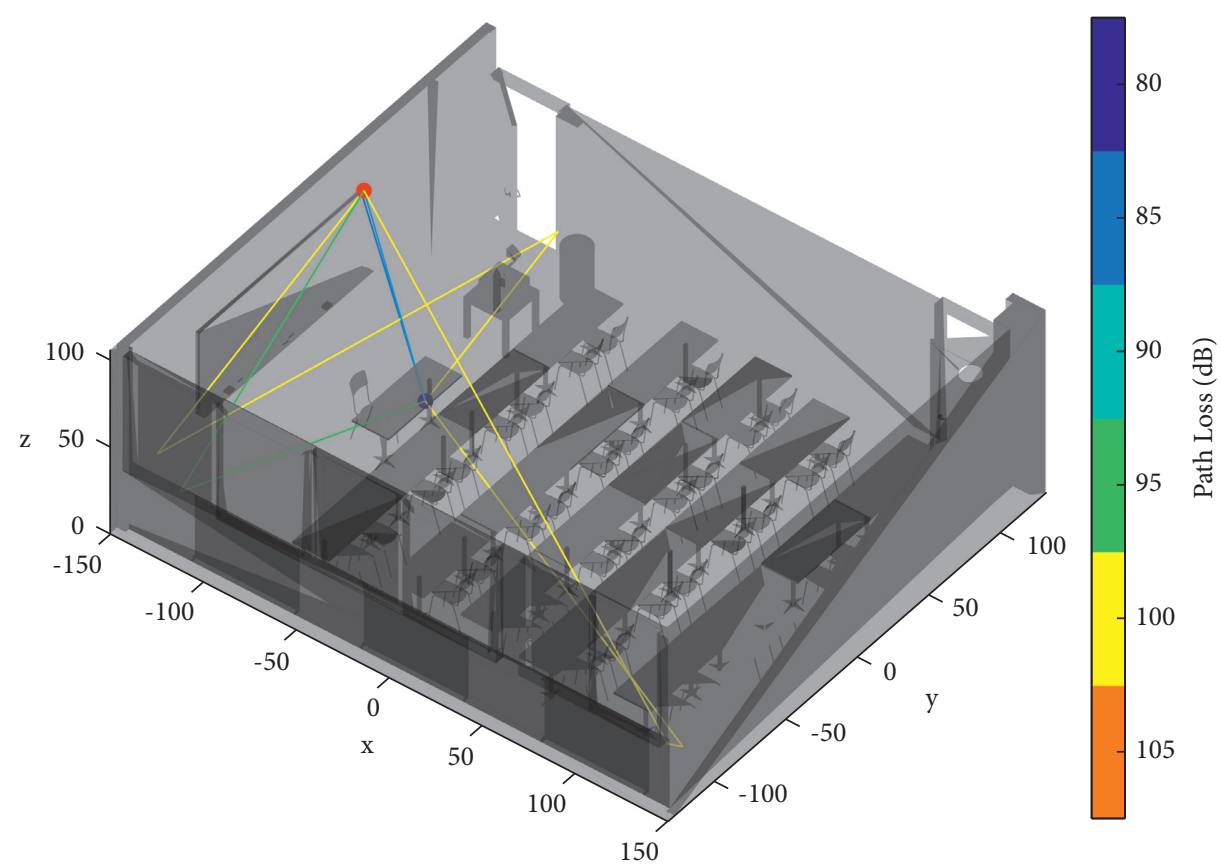

(b)

FIGURE 11: Indoor intelligent ray tracing. (a) At $28 \mathrm{GHz}$; (b) at $60 \mathrm{GHz}$.

TABLE 3: Rays attributes for indoor ray tracing at $28 \mathrm{GHz}$.

\begin{tabular}{|c|c|c|c|c|c|}
\hline Ray number & 1 & 2 & 3 & 4 & 5 \\
\hline Frequency $(\mathrm{GHz})$ & \\
\hline Coordinate system & \multicolumn{5}{|c|}{ Cartesian } \\
\hline Transmitter & \multicolumn{5}{|c|}{$[-144.414 ; 37.593 ; 79.0609]$} \\
\hline Receiver location & \multicolumn{5}{|c|}{$[-94.898 ; 16.208 ; 0.763]$} \\
\hline Propagation path & LoS & LoS & NLoS & NLoS & NLoS \\
\hline Reflection location & {$[10 ; 10 ; 0]$} & $\begin{array}{c}{[-117.91 ;-125.3} \\
37.161]\end{array}$ & $\begin{array}{c}{[-145.41,-110.15 ; 37.17} \\
22.54 ; 77.54,23.94]\end{array}$ & $\begin{array}{c}{[-128.74,-104.99 ;-127.15} \\
122.38 ; 54.27,16.73]\end{array}$ & $\begin{array}{c}{[144.41,150.21 ;-125.31,} \\
-122.04 ; 37.16,36.32]\end{array}$ \\
\hline $\begin{array}{l}\text { Propagation delay } \\
(\mu \mathrm{s})\end{array}$ & 0.3171 & 1.06 & 0.206 & 1.76 & 2.0835 \\
\hline $\begin{array}{l}\text { Propagation } \\
\text { distance }(\mathrm{m})\end{array}$ & 9.5 & 31.8 & 9.6 & 52.86 & 62.46 \\
\hline Angle of departure & $\begin{array}{c}{[-23.35} \\
-55.43]\end{array}$ & {$[-80.76 ;-14.24]$} & {$[-157.45 ;-54.53]$} & {$[-84.56 ;-8.51]$} & {$[-29.42 ;-7.20]$} \\
\hline Angle of arrival & $\begin{array}{l}{[156.64 ;} \\
55.43]\end{array}$ & $\begin{array}{c}{[-99.238} \\
14.244]\end{array}$ & {$[157.45 ; 54.53]$} & {$[95.43 ; 8.51]$} & {$[-29.42 ; 7.20]$} \\
\hline $\begin{array}{l}\text { Number of } \\
\text { reflections }\end{array}$ & 0 & 1 & 2 & 2 & 2 \\
\hline Path loss $(\mathrm{dB})$ & 80.95 & 91.44 & 81.05 & 95.85 & 97.31 \\
\hline Phase shift $\left({ }^{\circ}\right)$ & 0.0105 & 0.1073 & 5.490 & 2.0279 & 4.348 \\
\hline
\end{tabular}


TABLE 4: Rays attributes for indoor ray tracing at $60 \mathrm{GHz}$.

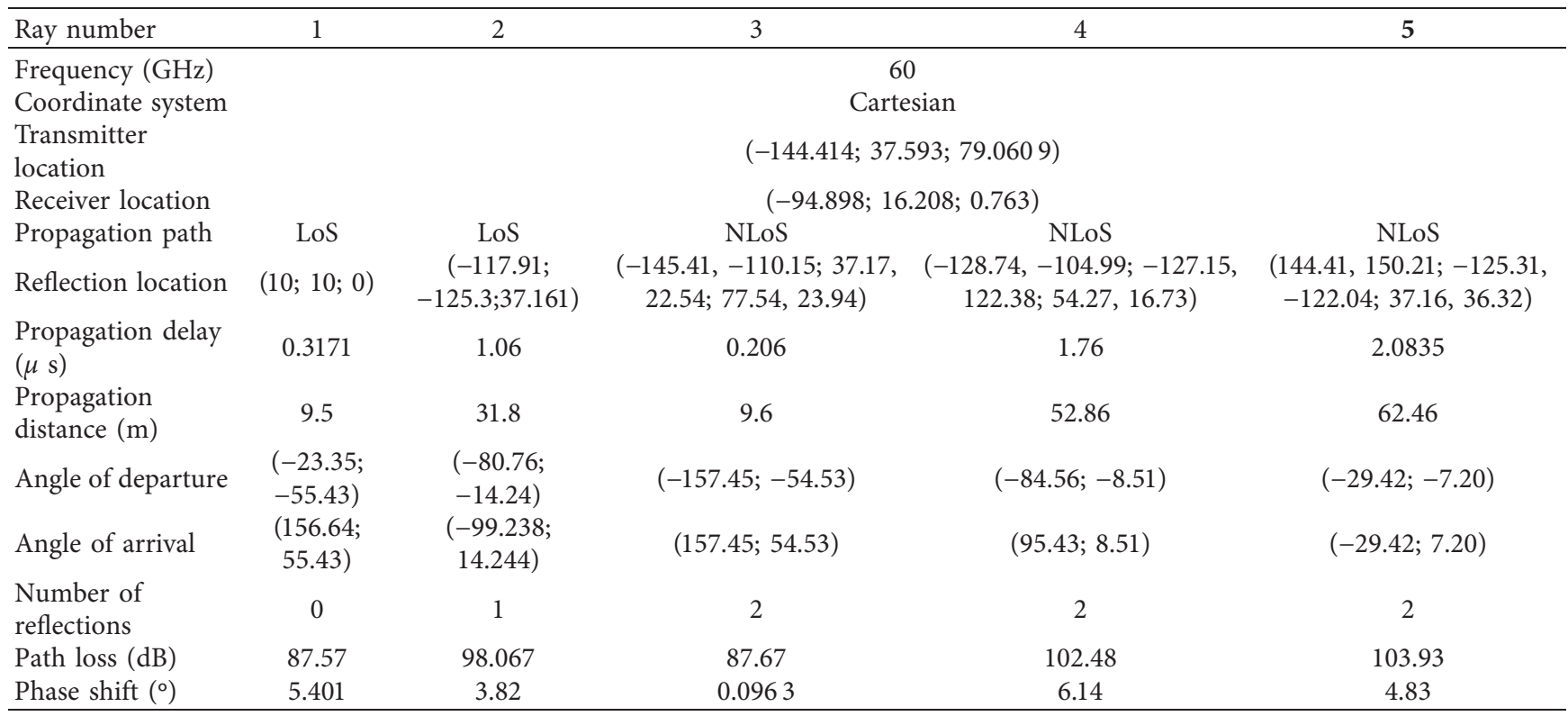

\section{Conclusion}

In this paper, the outdoor to outdoor and indoor to indoor characteristics of the path loss with increasing the mmWave frequencies considering the network forensic with the help of the dominant path algorithm are studied. In the first approach, a conventional approach of the empirical and statistical analysis of path loss is adopted. Three different models including ABG, $\mathrm{CI}$, and FI are discussed. The conclusion of the models is done that close in is better for indoor as well as outdoor scenarios. In the second approach, a software-based method is applied. Raytracing simulations indicate that the outdoor RSS level has a mean difference of around $8 \mathrm{dBm}-10 \mathrm{dBm}$ between $28 \mathrm{GHz}$ and $60 \mathrm{GHz}$. This is due to the fact that, on the one hand, the huge gap between mmWave frequencies does not affect the power of the signal in small cell environments. On the other hand, this is the sign of low penetration, low signal diffraction, and very high free-space losses. Similarly, in indoor environment, the difference between the path loss is due to the two different transmitting frequencies which is promising in a way that increasing the frequency in indoor environment results in an increase in the path loss by $5 \mathrm{~dB}$ for every $10 \mathrm{GHz}$. In the future, we would like to investigate radio network forensic for artificial intelligence-aided wireless systems.

\section{Data Availability}

No data were used to support this study.

\section{Conflicts of Interest}

The authors declare that they have no conflicts of interest.

\section{Acknowledgments}

This research was supported by Taif University Researchers Supporting Project number (TURSP-2020/314), Taif University, Taif, Saudi Arabia.

\section{References}

[1] S. Chen, K. Zeng, and P. Mohapatra, "Efficient data capturing for network forensics in cognitive radio networks," IEEE/ ACM Transactions on Networking, vol. 22, no. 6, pp. 19882000, 2013.

[2] C. Meisch, "FCC adopts rules to facilitate next generation wireless technologies," 2021, https://www.fcc.gov/document/ fcc-adopts-rules-facilitate-next-generation-wirelesstechnologies.

[3] U. R. Kamboh, Q. Yang, M. Qin, and S. Rauf, "Uncertainty cost analysis of heterogeneous wireless network based on loan repayment approach," in Proceedings of the 2017 Nineth International Conference on Advanced Infocomm Technology (ICAIT), pp. 170-175, Chengdu, China, November 2017.

[4] J. Lloret, J. J. López, C. Turró, and S. Flores, "Afast Design Model for Indoor Radio Coverage in the $2.4 \mathrm{GHz}$ Wireless LAN," in Proceedings of the Wireless CommunicationSystems, 2004, Firstst International Symposium on, Mauritius, pp. 408-412, Mauritius, September 2004.

[5] U. R. Kamboh, U. Ullah, S. Khalid, U. Raza, C. Chakraborty, and F. Al-Turjman, "Path Loss Modelling at $60 \mathrm{GHz}$ mmWave Based on Cognitive 3D ray Tracing Algorithm in 5G," PeerTo-Peer Networking and Applications, vol. 14, no. 8, 2021.

[6] W. Khawaja, O. Ozdemir, Y. Yapici, F. Erden, M. Ezuma, and I. Guvenc, "Coverage Enhancement for NLOS Mmwave Links Using Passive Reflectors,” 2019, https://arxiv.org/pdf/1905. 04794.pdf.

[7] W. Khawaja, O. Ozdemir, Y. Yapici, I. Guvenc, and Y. Kakishima, "Coverage enhancement for mmWave communications using passive reflectors," in Proceedings of the IEEE Global Symp. Millimeter Waves (GSMM), pp. 1-6, Boulder, CO, USA, May 2018.

[8] M. Kim, J. Liang, J. Lee, J. Park, B. Park, and H. K. Chung, "Investigating the effect of antenna beamwidth on millimeterwave channel characterization," in Proceedings of the IEEE URSI Asia-Pacific Radio Sci. Conf. (URSI AP-RASC), pp. 1-4, Seoul, Republic of Korea, August 2016.

[9] D. M. Tuan, Y. Cheon, Y. Aoki, and Y. Kim, "Performance comparison of millimeter-wave communications system with 
different antenna beamwidth," in Proceedings of the IEEE European Conf. Antennas Propag. (EuCAP), pp. 1-5, Davos, Switzerland, April 2016.

[10] H. Cheng, S. Ma, and H. Lee, "CNN-based mmWave path loss modeling for fixed wireless access in suburban scenarios," IEEE Antennas and Wireless Propagation Letters, vol. 19, no. 10, pp. 1694-1698, 2020.

[11] N. Kuno, W. Yamada, M. Sasaki, and Y. Takatori, "Convolution neural network for prediction method of path loss characteristics considering diffraction and reflection in an open-square environment," in Proceedings of the . URSI AsiaPacifc Radio Sci. Conf. (AP-RASC), pp. 1-3, New Delhi, India, March 2019.

[12] E. I. Adegoke, E. Kampert, and M. D. Higgins, "Empirical indoor path loss models at $3.5 \mathrm{GHz}$ for $5 \mathrm{G}$ communications network planning," in Proceedings of the. Int.Conf. UK-China Emerg. Technol. (UCET), pp. 1-4, Glasgow, UK, August 2020.

[13] M. C. Lawton and J. P. McGeehan, "The application of a deterministic ray launching algorithm for the prediction of radio channel characteristics in small-cell environments," IEEE Transactions on Vehicular Technology, vol. 43, no. 4, pp. 955-969, 1994.

[14] A. Al-Samman, T. Rahman, M. Hindia, A. Daho, and E. Hana, "Path loss model for outdoor parking environments at 28 $\mathrm{GHz}$ and $38 \mathrm{GHz}$ for $5 \mathrm{G}$ wireless networks," Symmetry, vol. 10, no. 12, p. 672, 2018.

[15] S. Li, Y. Liu, L. Lin, D. Sun, S. Yang, and X. Sun, "Simulation and modeling of millimeter-wave channel at $\mathrm{GHz}$ in indoor environment for 5G wireless communication system," in Proceedings of the 2018 IEEE International Conference on Computational Electromagnetics (ICCEM), pp. 1-3, March 2018.

[16] G. R. Maccartney, T. S. Rappaport, M. K. Samimi, and S. Sun, "Millimeter-wave omnidirectional path loss data for small cell 5G channel modeling," IEEE Access, vol. 3, pp. 1573-1580, 2015.

[17] A. M. Al-Samman, M. H. Azmi, Y. A. Al-Gumaei et al., "Millimeter wave propagation measurements and characteristics for 5G system," Applied Sciences, vol. 10, no. 1, p. 335, 2020.

[18] A. M. Al-Samman, T. A. Rahman, T. Al-Hadhrami et al., "Comparative study of indoor propagation model below and above $6 \mathrm{GHz}$ for $5 \mathrm{G}$ wireless networks," Electronics, vol. 8, no. 1, p. 44, 2019.

[19] I. Cuinas, M. G. Sanchez, A. Feys, W. Debaenst, and J. Verhaevert, "Indoor path loss variations with frequency and visibility conditions at $3.5 \mathrm{GHz}$ band," in Proceedings of the 2019 IEEE International Symposium on Antennas and Propagation and USNC-URSI Radio Science Meeting, pp. 2069-2070, Atlanta, GA, USA, July 2019.

[20] N. Khalid, N. A. Abbasi, and O. B. Akan, "Statistical characterization and analysis of low- $\mathrm{THz}$ communication channel for 5G Internet of Things," Nano Commun. Netw.vol. 22, Article ID 100258, 2019.

[21] F. Qamar, M. N. Hindia, K. Dimyati et al., "Investigation of future 5G-IoT millimeter-wave network performance at 38 $\mathrm{GHz}$ for urban microcell outdoor environment," Electronics, vol. 8, no. 5, p. 495, 2019.

[22] Y. Zhou, X. Sun, P. Zhang, H. Wang, Z. Hu, and H. Wang, "Multi-frequency millimeter-wave large-scale path loss characterization for indoor environment," in Proceedings of the 2019 International Symposium on Antennas and Propagation (ISAP), pp. 1-3, Xi'an, China, October 2019.
[23] J. Wen, Y. Zhang, G. Yang, Z. He, and W. Zhang, "Path loss prediction based on machine learning methods for aircraft cabin environments," IEEE Access, vol. 7, Article ID 159251, 2019.

[24] H. Cheng, S. Ma, H. Lee, and M. Cho, "Millimeter wave path loss modeling for $5 \mathrm{G}$ communications using deep learning with dilated convolution and attention," IEEE Access, vol. 9, Article ID 62867, 2021.

[25] N. Kuno and Y. Takatori, "Prediction method by deeplearning for path loss characteristics in an open-square environment," In Proc. Int. Symp. Antennas Propag. (ISAP), pp. 1-2, 2018.

[26] A.-Y. Hsiao, C.-F. Yang, T.-S. Wang, I. Lin, and W.-J. Liao, "Ray tracing simulations for millimeter wave propagation in 5G wireless communications," in Proceedings of the 2017 IEEE International Symposium on Antennas and Propagation \& USNC/URSI National Radio Science Meeting, pp. 1901-1902, San Diego, CA, USA, July 2017.

[27] C. Ge, Y. Zhang, and X. Jiang, "Simulation and analysis of 28 $\mathrm{GHz}$ millimeter-wave propagation characteristics in typical residential house environment," in Proceedings of the 2018 11th UK-Europe-China Workshop on Millimeter Waves and Terahertz Technologies (UCMMT), vol. 1, pp. 1-3, HangZhou, China, September 2018.

[28] K. Kitao, A. Benjebbour, T. Imai, Y. Kishiyama, M. Inomata, and Y. Okumura, "5G system evaluation tool," in Proceedings of the 2018 IEEE International Workshop on Electromagnetics: Applications and Student Innovation Competition (iWEM), pp. 1-2, Nagoya, Japan, August 2018.

[29] F. Hossain, T. Geok, T. Rahman et al., "An efficient 3-D ray tracing method: prediction of indoor radio propagation at 28 GHz in 5G network," Electronics, vol. 8, no. 3, p. 286, 2019.

[30] C.-F. Chang-Fa Yang, B.-C. Boau-Cheng Wu, and C.-J. Chuen-Jyi Ko, "A ray-tracing method for modeling indoor wave propagation and penetration," IEEE Transactions on Antennas and Propagation, vol. 46, no. 6, pp. 907-919, 1998.

[31] A. E. Shaikh, F. Majeed, M. Zeeshan, T. Rabbani, and I. Sheikh, "Efficient implementation of deterministic 3-D ray tracing model to predict propagation losses in indoor environments," in Proceedings of the 13th IEEE International Symposium on Personal, Indoor and Mobile Radio Communications, pp. 1208-1212, Lisboa, Portugal, September 2002.

[32] U. Ullah, U. R. Kamboh, F. Hossain, and M. Danish, "Outdoor-to-Indoor and indoor-to-indoor propagation path loss modeling using smart $3 \mathrm{D}$ ray tracing algorithm at $28 \mathrm{GHz}$ mmWave," Arabian Journal for Science and Engineering, vol. 45, pp. 1-10, 2020.

[33] D. Murugesan and T. R. Rao, "Indoor corridor radio propagation characteristics at $60 \mathrm{GHz}$ for wireless communications," in Proceedings of the 2012 3rd International Conference on Computing, Communication and Networking Technologies (ICCCNT'12), pp. 1-5, Coimbatore, India, July 2012.

[34] A. Karstensen, W. Fan, I. Carton, and G. F. Pedersen, "Comparison of ray tracing simulations and channel measurements at mmwave bands for indoor scenarios," in Proceedings of the 2016 10th European Conference on Antennas and Propagation (EuCAP), pp. 1-5, Davos, Switzerland, April 2016.

[35] S. Helhel, "Comparison of 900 and $1800 \mathrm{MHz}$ indoor propagation deterioration," IEEE Transactions on Antennas and Propagation, vol. 54, no. 12, pp. 3921-3924, 2006.

[36] J. J. P. C. Rodrigues, S. Jabbar, M. Abdallah, C. Verikoukis, and M. Guizani, "Future communication trends toward 
internet of things services and applications," IEEE Wireless Communications, vol. 26, no. 6, pp. 6-8, 2019.

[37] F. A. Rodríguez-Corbo, L. Azpilicueta, M. Celaya-Echarri et al., "Deterministic 3D ray-launching millimeter wave channel characterization for vehicular communications in urban environments," Sensors, vol. 20, no. 18, p. 5284, 2020.

[38] G. R. Maccartney, T. S. Rappaport, S. Sun, and S. Deng, "Indoor office wideband millimeter-wave propagation measurements and channel models at 28 and $73 \mathrm{GHz}$ for ultradense 5G wireless networks," IEEE Access, vol. 3, pp. 23882424, 2015.

[39] S. Sun, T. A. Thomas, T. S. Rappaport, H. Nguyen, I. Z. Kovacs, and I. Rodriguez, "Path Loss, Shadow Fading, and Line-Of-Sight Probability Models for 5G Urban MacroCellular Scenarios," in Proceedings of the 2015 IEEE Globecom Workshops (GC Wkshps), San Diego, CA, USA, December 2015.

[40] A. M. Al-Samman, T. A. Rahman, M. H. Azmi, M. N. Hindia, I. Khan, and E. Hanafi, "Statistical modelling and characterization of experimental $\mathrm{mm}$-wave indoor channels for future 5G wireless communication networks," PLoS One, vol. 11, no. 9, Article ID e0163034, 2016.

[41] Z. Yun and M. F. Iskander, "Ray tracing for radio propagation modeling: principles and applications," IEEE Access, vol. 3, pp. 1089-1100, 2015.

[42] M. F. Iskander and Z. Zhengqing Yun, "Propagation prediction models for wireless communication systems," IEEE Transactions on Microwave Theory and Techniques, vol. 50, no. 3, pp. 662-673, 2002.

[43] G. Durgin, N. Patwari, and T. S. Rappaport, "An advanced 3D ray launching method for wireless propagation prediction," in Proceedings of the 1997 IEEE 47th Vehicular Technology Conference. Technology in Motion, vol. 2, pp. 785-789, Phoenix, AZ, USA, May 1997.

[44] D. He, B. Ai, K. Guan, L. Wang, Z. Zhong, and T. Kurner, "The design and applications of high-performance ray-tracing simulation platform for $5 \mathrm{G}$ and beyond wireless communications: a tutorial," IEEE Communications Surveys \& Tutorials, vol. 21, no. 1, pp. 10-27, 2019.

[45] V. Degli-Esposti, D. Guiducci, A. de’Marsi, P. Azzi, and F. Fuschini, "An advanced field prediction model including diffuse scattering," IEEE Transactions on Antennas and Propagation, vol. 52, no. 7, pp. 1717-1728, 2004.

[46] R. Luebbers, "Finite conductivity uniform GTD versus knife edge diffraction in prediction of propagation path loss," IEEE Transactions on Antennas and Propagation, vol. 32, no. 1, pp. 70-76, 1984.

[47] O. Franek, S. Zhang, K. Olesen, P. C. F. Eggers, C. Byskov, and G. F. Pedersen, "Numerical modeling of ultrawideband propagation along a wind turbine blade," IEEE Transactions on Antennas and Propagation, vol. 66, no. 12, pp. 6570-6579, 2018.

[48] M. S. L. Mocker, M. Schiller, R. Brem et al., "Combination of a full-wave method and ray tracing for radiation pattern simulations of antennas on vehicle roofs," in Proceedings of the Nineth Eur. Conf. Antennas Propag. (EuCAP), pp. 1-5, Lisbon, Portugal, April 2015.

[49] S. Jabbar, H. Lloyd, M. Hammoudeh, B. Adebisi, and U. Raza, "Blockchain-enabled supply chain: analysis, challenges, and future directions," Multimedia Systems, vol. 27, no. 4, pp. 787-806, 2021. 